\title{
Doxorubicin combined with Notch1-targeting siRNA for the treatment of gastric cancer
}

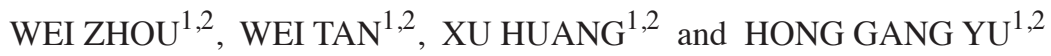 \\ ${ }^{1}$ Department of Gastroenterology; ${ }^{2}$ Hubei Key Laboratory of Digestive System Disease, \\ Renmin Hospital of Wuhan University, Wuhan, Hubei 430060, P.R. China
}

Received September 30, 2017; Accepted March 1, 2018

DOI: $10.3892 / \mathrm{ol} .2018 .9039$

\begin{abstract}
Notch1, a transmembrane receptor that has a notable role in gastric cancer (GC) as an oncogene, has been reported to be involved in doxorubicin resistance. Thus, Notch1 is a potential therapeutic target for GC. In the present study, the protein levels of Notch1 intracellular domain (NICD; a marker of Notch1 activation) in human GC cell lines and tumor tissues was measured by western blotting. Next, the effects of Notch1 depletion in SGC7901 cells were evaluated. Finally, the efficacy of Notch1 small interfering RNA (siRNA) combined with doxorubicin therapy for GC was examined in vitro and in vivo. The results revealed that NICD levels were high in GC cells, and that the inhibition of NICD by transfection with Notch1 siRNA induced apoptosis and inhibited proliferation. Ectopic downregulation of Notch1 expression enhanced the sensitivity of GC tumors to doxorubicin, which suppressed the development of GC. These data demonstrated that Notch1 was a significant regulator of cell proliferation and apoptosis in GC. Thus, the combination of doxorubicin with Notch1 siRNA is a potential strategy for the treatment of GC.
\end{abstract}

\section{Introduction}

Gastric cancer (GC) is a major health problem worldwide; it is associated with high mortality rates, particularly in East Asian countries, including China (1). Although the survival of patients with GC has been improved by recent surgical advances, the long-term survival rate of patients following surgical resection remains unsatisfactory, owing to high rates of recurrence and metastasis (2). Chemotherapy is one of the first choices for patients with unresectable locally advanced or metastatic GC (3). Doxorubicin (DOX) is an anthracycline antibiotic that is widely used in chemotherapy. It is a cell-cycle non-specific antitumor drug that exerts its action mainly by

Correspondence to: Dr Wei Zhou, Department of Gastroenterology, Renmin Hospital of Wuhan University, 238 Jiefang Road, Wuhan, Hubei 430060, P.R. China

E-mail: 3038462163@qq.com

Key words: Notch1, doxorubicin, resistant, apoptosis, gastric cancer inducing apoptosis, autophagy and necrosis (4). However, in patients with GC, DOX exhibits poor clinical outcomes and causes certain adverse effects (5). Therefore, combination treatment strategies that enhance the efficiency of DOX are required.

Notch1 is a multifunctional transmembrane receptor that belongs to the highly conserved Notch family of proteins. Previous studies revealed that the Notch1 signaling pathway is activated by the interaction of Notch1 receptor with its ligands, Jagged1, Jagged2, Delta1, Delta3 and Delta4 (6,7). First, Notch1 is cleaved at the Golgi apparatus by furin and translocated to the plasma membrane. Next, it is bound by its ligands and cleaved by proteases, releasing the Notch1 intracellular domain (NICD), which translocates to the nucleus. Finally, the NICD binds to its downstream target genes to exert various biological effects (8). In human cancer, Notch1 functions as either a tumor-suppressor or an oncogene based on cellular context (9). For example, Notch1 is activated in several cancer types, including lymphoma, colon cancer, non-small cell lung cancer and GC, in which it functions as an oncogene (10-13). By contrast, opposite functions are observed in neuroendocrine tumors (NETs), cervical cancer, and prostate cancer $(14,15)$. Thus, the Notch1 signaling pathway regulates cell proliferation, differentiation and apoptosis during tumor development in a variety of contexts and is a potential therapeutic target for GC.

Notch1 was previously reported to reduce doxorubicin resistance in small cell lung carcinoma cells (16). However, there have been few reports on the association of Notch1 with the sensitivity of GC to doxorubicin (17-19). Thus, the aim of the present study was to investigate the role of Notch1 in GC and the efficacy of Notch1 small interfering RNA (siRNA) combined with doxorubicin therapy for GC treatment, which could provide a novel therapeutic approach for GC.

\section{Materials and methods}

Tissue specimens. Matched tissue samples of primary GC and adjacent normal mucosa were collected from 26 patients with GC that underwent surgical resection at the Renmin Hospital of Wuhan University (Wuhan, China) between January 2012 and April 2016. None of the study patients had received chemotherapy or radiation therapy prior to tumor resection. Each case was reviewed by two experienced histopathologists. 
All tissues were snap-frozen in liquid nitrogen and stored at $-80^{\circ} \mathrm{C}$ until use. Informed consent was obtained from all patients in accordance with the Declaration of Helsinki (20), and all human tissue procedures were approved by the Ethical Committee on Clinical Research of Renmin Hospital of Wuhan University (AF SOP/3.6-01/5.1).

Immunohistochemistry. Paired GC and normal mucosal specimens were embedded in paraffin and cut into $4-\mu \mathrm{m}$-thick sections and mounted on glass slides. Following mounting, they were kept in an oven at $70^{\circ} \mathrm{C}$. Sections were then deparaffinized with xylene twice for $5 \mathrm{~min}$ and rehydrated with ethanol gradient $(100,95,90,80$ and $70 \%$, diluted in double distilled water) for $2 \mathrm{~min}$ at room temperature. The sections were blocked with $1 \% \mathrm{H}_{2} \mathrm{O}_{2}$ at room temperature for $30 \mathrm{~min}$ against the endogenous peroxidase activity, treated for antigen retrieval by microwave in Citrate buffer $(10 \mathrm{mmol} / \mathrm{l}, \mathrm{pH} 6.0$, Beijing Solarbio Science \& Technology Co., Ltd., Beijing, China) for $10 \mathrm{~min}$, washed with PBS and incubated with goat serum (Gibco; Thermo Fisher Scientific, Inc., Waltham, MA, USA) blocking solution for $1 \mathrm{~h}$ at room temperature. The blocked sections were incubated with the primary antibodies against NICD (cat. no. 3608; Cell Signaling Technology, Inc., Danvers, MA, USA) overnight at $4^{\circ} \mathrm{C}$ at a dilution of 1:50 and then incubated with biotin-labeled anti-rabbit IgG $(1: 1,000$; cat. no. ab6720; Abcam, Cambridge, MA, USA) for $1 \mathrm{~h}$ at room temperature. A total of 10 randomly selected fields under x400 magnification were observed under a light microscope (Carl Zeiss AG, Oberkochen, Germany).

Cell culture. The human gastric carcinoma cell lines SGC7901, BGC823, MKN45, HGC27 and the human gastric mucosal epithelial cell line GES-1 were purchased from the Type Culture Collection of the Chinese Academy of Sciences (Shanghai, China). Cells were cultured in RPMI-1640 (Gibco; Thermo Fisher Scientific, Inc.) supplemented with $10 \%$ heat-inactivated fetal bovine serum (FBS; Gibco; Thermo Fisher Scientific, Inc.), penicillin $(100 \mathrm{U} / \mathrm{ml})$, and streptomycin (100 $\mu \mathrm{g} / \mathrm{ml}$; Biosharp, Hefei, China, http://www.biosharp. cn/index.asp) in a humidified atmosphere containing $5 \% \mathrm{CO}_{2}$ at $37^{\circ} \mathrm{C}$.

Transfection. SGC7901 cells were seeded in 6-well low-attachment plates $\left(10^{3}\right.$ cells $\left./ \mathrm{cm}^{2}\right)$ and transfected with final concentration $25 \mathrm{nmol} / 1$ control siRNA (catalog no. D-001810-10-20, On-TARGETplus Non-Targeting Pool; GE Healthcare Dharmacon, Inc., Lafayette, CO, USA) or Notch1 siRNA (5'-UGGACAUGA ACUAAUUAGU-3', 5'-GUCAUAGCUCUCAGAUUUC-3', 5'-UCUGAUGAC ACCAUUAAUA-3', 5'-UCAGGUUGUUGGAUUGGUG-3') (ON-TARGETplus SMARTpool; Dharmacon, Inc.) using Lipofectamine 2000 reagent (Invitrogen; Thermo Fisher Scientific, Inc.) according to the manufacturer's protocol. Following culturing for $48 \mathrm{~h}$ at $37^{\circ} \mathrm{C}$, transfection efficiency was assessed by western blotting, and then the cells were cultured for other experiments.

Western blot assay. Control and Notch1 siRNA-transfected SGC7901 cells $48 \mathrm{~h}$ after transfected were applied to western blot analysis. Control and Notch1 siRNA-transfected
SGC7901 cells combined with or without $0.3 \mu \mathrm{M}$ doxorubicin for $24 \mathrm{~h}$ were applied in western blot analysis (21). Briefly, cells were washed with PBS ( $\mathrm{pH} 7.4)$ and lysed by incubation in lysis buffer (20 mM Tris-HCl, pH 7.4, $150 \mathrm{mM} \mathrm{NaCl}, 0.5 \%$ Nonidet P-40, $1 \mathrm{mM}$ EDTA, $50 \mu \mathrm{g} / \mathrm{ml}$ leupeptin, $30 \mu \mathrm{g} / \mathrm{ml}$ aprotinin and $1 \mathrm{mM}$ PMSF) for $30 \mathrm{~min}$ at room temperature. The protein concentration were detected by BCA Protein assay kit (Beyotime Institute of Biotechnology, Haimen, China). Next, whole-cell lysates (40 $\mu \mathrm{g}$ per lane) were loaded on and separated by $12.5 \%$ SDS-PAGE. The proteins in the gel were then transferred to polyvinylidene fluoride membranes (EMD Millipore, Billerica, MA, USA) with a wet transfer system (Bio-Rad Laboratories, Inc., Hercules, CA, USA). Next, the membrane was blocked with $10 \%$ non-fat dry milk in Tris-buffered saline with $0.05 \%$ Tween-20 (TBST; pH 8.0), and then incubated with primary antibodies against Notch1, NICD (cat. nos. 4380 and 3608, 1:500, respectively; both Cell Signaling Technology, Inc.), cyclin D1, tumor protein p53 (hereafter p53), B-cell lymphoma-2 (Bcl-2), and $\beta$-actin (cat. nos. sc-8396, 1:500; sc-17846, 1:200; sc-509, 1:500; and sc-8432, 1:500, respectively; Santa Cruz Biotechnology, Inc., Dallas, TX, USA) overnight at $4^{\circ} \mathrm{C}$. The blot was then incubated with either the appropriate horseradish peroxidase goat anti-mouse or goat anti-rabbit IgG H\&L secondary antibodies (cat. nos. ab6728 and ab6721; 1:3,000; Abcam) for $2 \mathrm{~h}$ at room temperature. Positive antibody reactions were detected with an enhanced chemiluminescence system (GE Healthcare Life Sciences, Pittsburgh, PA, USA) and Hyperfilm X-ray film.

Growth inhibition. Cell growth inhibition by doxorubicin and Notch1 siRNA was assessed by the MTT assay. SGC7901 cells transfected with control or Notch1 siRNA were seeded into 96 -well plates $\left(6.0 \times 10^{3}\right.$ cells/well) and allowed to attach overnight. Following attachment, freshly prepared doxorubicin (Enzo Life Sciences, Inc., Farmingdale, NY, USA) was added at different concentrations $(0.03,0.3$, and $3 \mu \mathrm{M})$. Next, the viability of these cells at three developmental time points $(12,24$, and $48 \mathrm{~h}$ ) was evaluated using Cell Proliferation kit I, according to the manufacturer's protocol (Roche Applied Science, Penzberg, Germany). Briefly, $500 \mathrm{mg} / 1 \mathrm{MTT}$ was added to each well and incubated for $4 \mathrm{~h}$ at $37^{\circ} \mathrm{C}$. Next, the samples were solubilized with $10 \%$ sodium dodecyl sulfate in $10 \mathrm{mmol} / 1 \mathrm{HCl}$ overnight at $37^{\circ} \mathrm{C}$ and the absorbance of each well at $570 \mathrm{~nm}$ was determined using a computer-controlled microtiter plate reader. All experiments were performed in triplicate and normalized to that of untreated SGC7901 cells.

Apoptosis analysis. Control and Notch1 siRNA-transfected cells were incubated with or without $0.3 \mu \mathrm{M}$ doxorubicin for $24 \mathrm{~h}$. Next, the cells were harvested and fixed with $2.5 \%$ glutaraldehyde for $30 \mathrm{~min}$ at $4^{\circ} \mathrm{C}$. Following washing of the cells twice with ice-cold PBS, apoptosis was assessed by a Becton Dickinson FACScan flow cytometry using the Annexin V-FITC Apoptosis kit (BioVision, Inc., Milpitas, CA, USA), data were analyzed with a flow cytometer using FlowJo software (version 7.6.2, TreeStar, Ashland, OR, USA). Caspase-3 activity was also evaluated with the Caspase-3 Colorimetric Assay kit (Nanjing Keygen Biotech Co., Ltd., Nanjing, China), according to the manufacturer's instructions. Briefly, the harvested cells were resuspended in $50 \mu \mathrm{l}$ 
A a
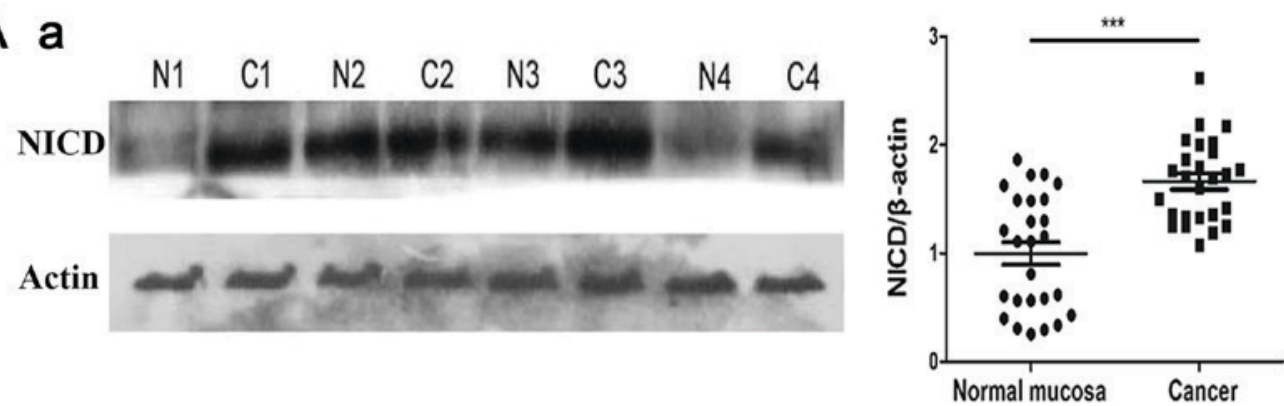

b
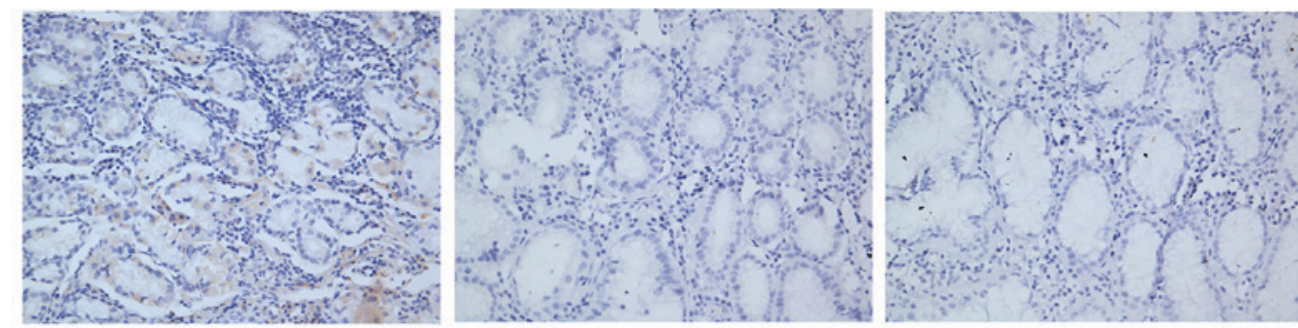

N1-NICD

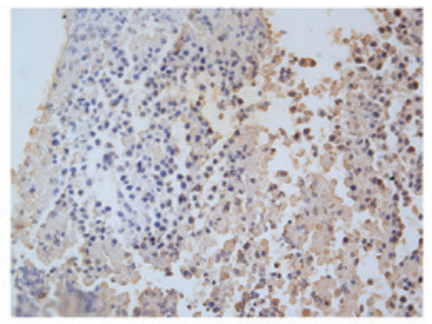

N2-NICD

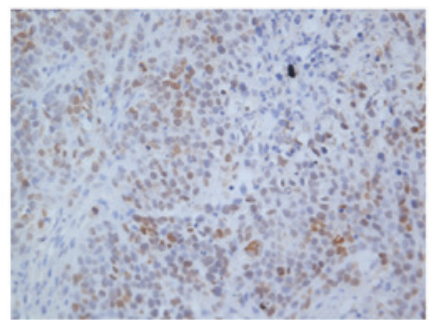

C2-NICD

N3-NICD

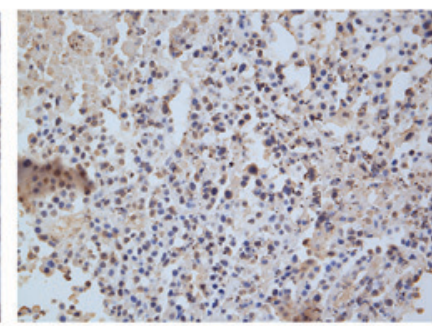

C3-NICD

B
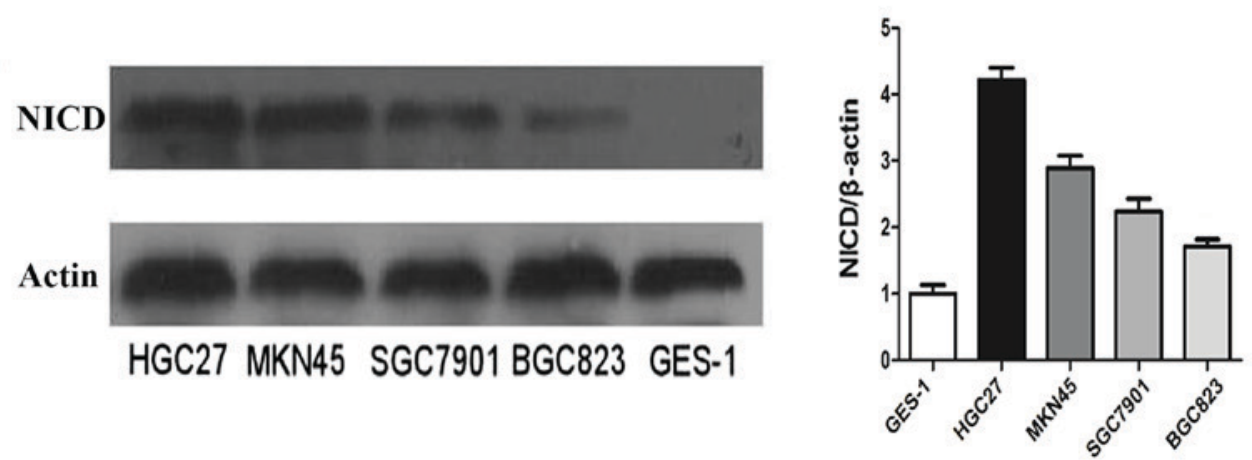

Figure 1. High levels of NICD in GC. (Aa) The levels of NICD in the representative paired tissues and the adjacent normal mucosa from patients with gastric cancer. (Ab) Immunohistochemical analysis of NICD in gastric cancer and paired normal mucosa (magnification, $\mathrm{x} 400$ ). (B) The levels of NICD in GC cell lines and normal gastric mucosa epithelial GES-1 cells. ${ }^{* * *} \mathrm{P}<0.001$. NICD, Notch1 intracellular domain; GC, gastric cancer; N, normal mucosa; C, cancer.

lysis buffer and incubated on ice for $30 \mathrm{~min}$. Next, the lysates were transferred to 96 -well plates, and caspase-3 activity was determined as described.

In vivo assay for tumor growth. Female BALB/c nude mice (15-20 g) were purchased from the Animal Research Center of Wuhan University (Wuhan, China). Mice were randomly divided into three groups of six mice each and maintained under SPF conditions $\left(26^{\circ} \mathrm{C}, 40-60 \%\right.$ humidity), supplied with free drinking water and food in a $12 \mathrm{~h}$ dark/light cycle. Next, $1 \times 10^{7}$ SGC7901 cells and control and Notch1 siRNA-transfected cells were separately injected into the flanks of mice. When the gastric tumors reached $100-150 \mathrm{~mm}^{3}$, the mice were treated with doxorubicin $(2 \mathrm{mg} / \mathrm{kg}$ ) every 4 days. Tumor growth was monitored by measuring the tumor volume, which was calculated according to the following formula: $\mathrm{V}=$ width $^{2} \mathrm{x}$ length $/ 2$. At the end of the experiment, the nude mice were sacrificed by cervical dislocation and the tumors were harvested for further analyses. All animal procedures were approved by the Ethical Committee on Animal Experimentation of Renmin Hospital of Wuhan University [WDRM (FU) no. 20151012], and were performed in accordance with the National Institutes of Health Guide for the Care and Use of Laboratory Animals (22).

TUNEL assay. The In Situ Apoptosis Detection kit (Roche Applied Science) was used to detect apoptosis in sections of 

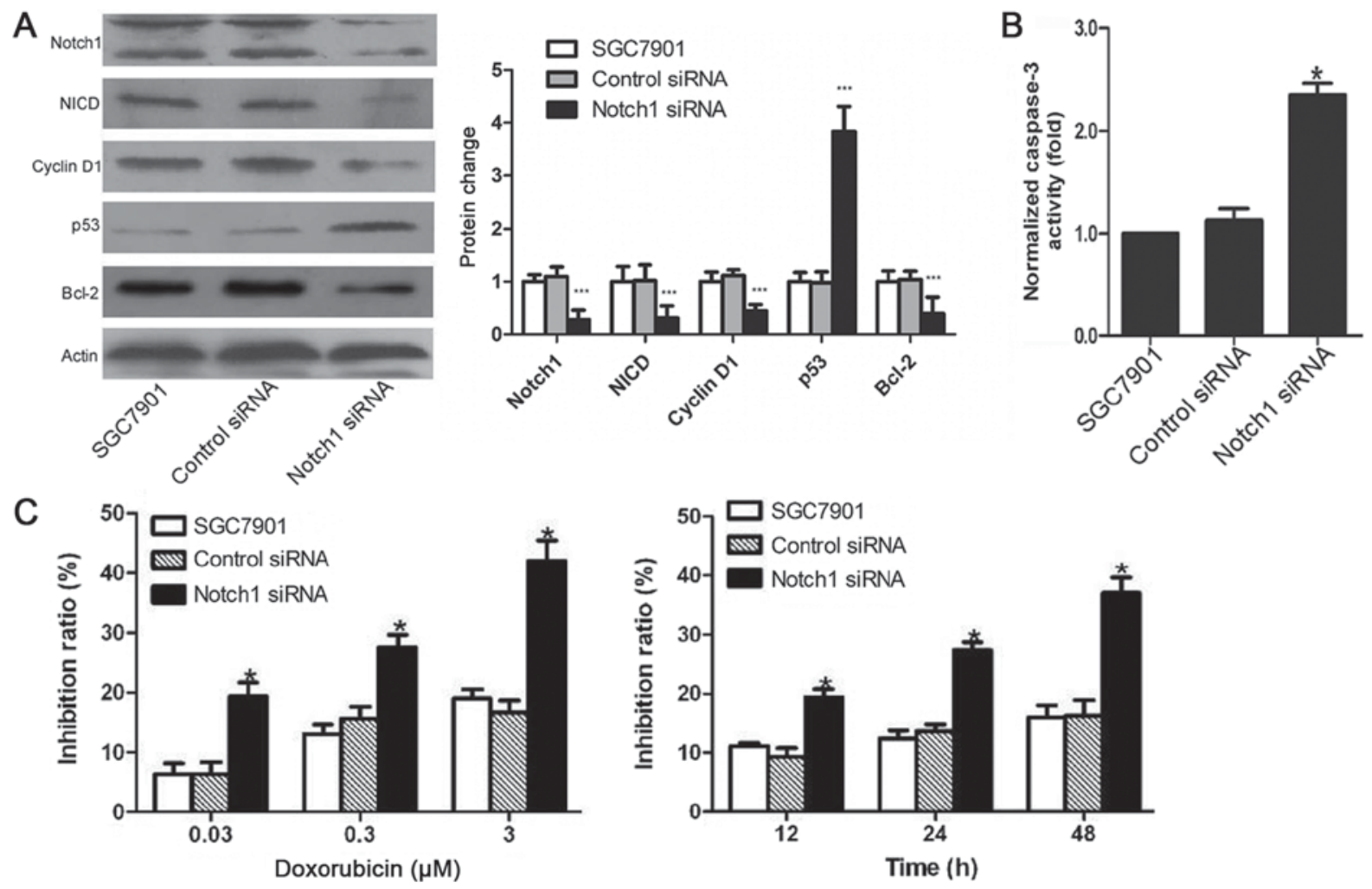

Figure 2. Effect of Notch1 knockdown on SGC7901. (A) Levels of Notch1, NICD and apoptosis-associated proteins following transfection were detected by western blotting. (B) Analysis of relative caspase-3 activity in SGC7901. (C) The rate of gastric cancer cells growth inhibition was measured by the MTT assay. ${ }^{*} \mathrm{P}<0.05$ and ${ }^{* * *} \mathrm{P}<0.001$ vs control siRNA. NICD, Notch1 intracellular domain; p53, tumor protein p53; Bcl-2, B-cell lymphoma-2.

tumor tissues from mice in the tumor growth assay. First, $4 \%$ paraformaldehyde fixed paraffin-embedded tumor sections were deparaffinized with dimethylbenzene twice for $5 \mathrm{~min}$ and rehydrated with an ethanol gradient $(100,95,90,80$ and $70 \%$, diluted in double distilled water) for $2 \mathrm{~min}$ at room temperature, and then incubated with proteinase $\mathrm{K}$ for $30 \mathrm{~min}$ at $37^{\circ} \mathrm{C}$. After washing in PBS three times, the sections were incubated with TUNEL reaction mixture at $37^{\circ} \mathrm{C}$ for $1 \mathrm{~h}$ and developed with diaminobenzidine. Finally, the sections were counterstained with hematoxylin. Positive cells were identified and counted (in three random fields per slide) under a light microscope (Carl Zeiss AG).

Statistical analysis. All experimental data were shown as the mean \pm standard error of the mean. The means of the different groups were compared using one-way analysis of variance followed by Duncan's multiple comparison statistical tests. All statistical analyses were performed using the SPSS 13.0 software (SPSS, Inc., Chicago, IL, USA). $\mathrm{P}<0.05$ was considered to indicate a statistically significant difference.

\section{Results}

Notch1 signaling is activated in GC. The levels of NICD were investigated in GC and paired normal gastric tissues, as well as in GC cells, by western blotting. NICD protein levels were detected in 54\% of normal (14/26) and 100\% of GC (26/26) tissues. However, the mean NICD protein level was significantly higher in GC tissues than in normal tissues $(\mathrm{P}<0.001$; Fig. 1A). In addition, immunohistochemistry confirmed that NICD levels in cancer tissues were higher than that in the normal mucosa. Consistent with this finding, NICD levels were elevated in GC cell lines (Fig. 1B). To investigate the biological characteristics of NICD in GC, SGC7901 cells that exhibited moderate levels of NICD were selected for further experimentation.

Effects of Notch1 siRNA on the proliferation and apoptosis of SGC7901 cells. To investigate the role of Notch1 in GC, the expression of Notch1 was inhibited in SGC7901 cells by transfecting with a specific siRNA. The results revealed that the depletion of Notch1 downregulated NICD, cyclin D1 and Bcl-2 levels, but upregulated those of p53 (Fig. 2A). Compared with untransfected and control siRNA-transfected SGC7901 cells, caspase-3 activity in Notch1 siRNA-transfected cells was $\sim 138 \%$ higher $(\mathrm{P}<0.05$; Fig. $2 \mathrm{~B})$. As expected, there was no significant difference in caspase-3 activity between untransfected and control siRNA-transfected SGC7901 cells. These results indicated that Notch1 may be a critical inhibitor of apoptosis in GC.

Notch1 siRNA enhances the doxorubicin sensitivity of GC. The effects of Notch1 siRNA and doxorubicin on cell growth were measured using an MTT assay. Doxorubicin significantly reduced the survival of SGC7901 cells treated with Notch1 siRNA, and this suppression was doxorubicin dose- and time-dependent (Fig. 2C). These results indicated that Notch1 siRNA inhibits SGC7901 cell growth and enhances the doxorubicin sensitivity of GC. 
A

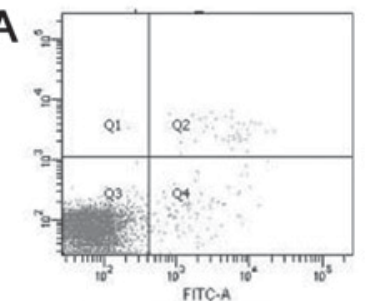

Control siRNA

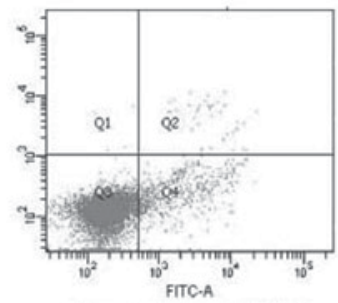

Notch1 siRNA

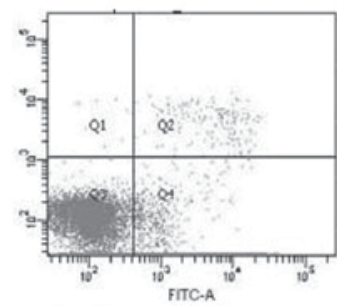

Control siRNA+Dox

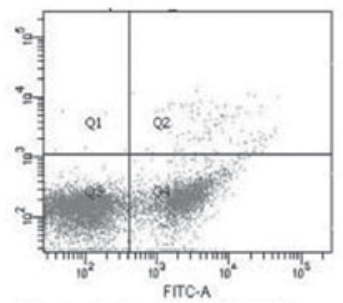

Notch1 siRNA+Dox

C

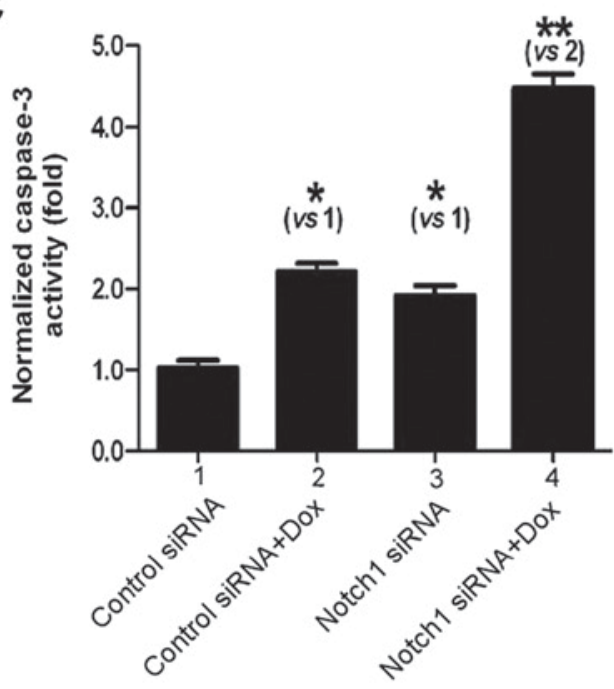

B
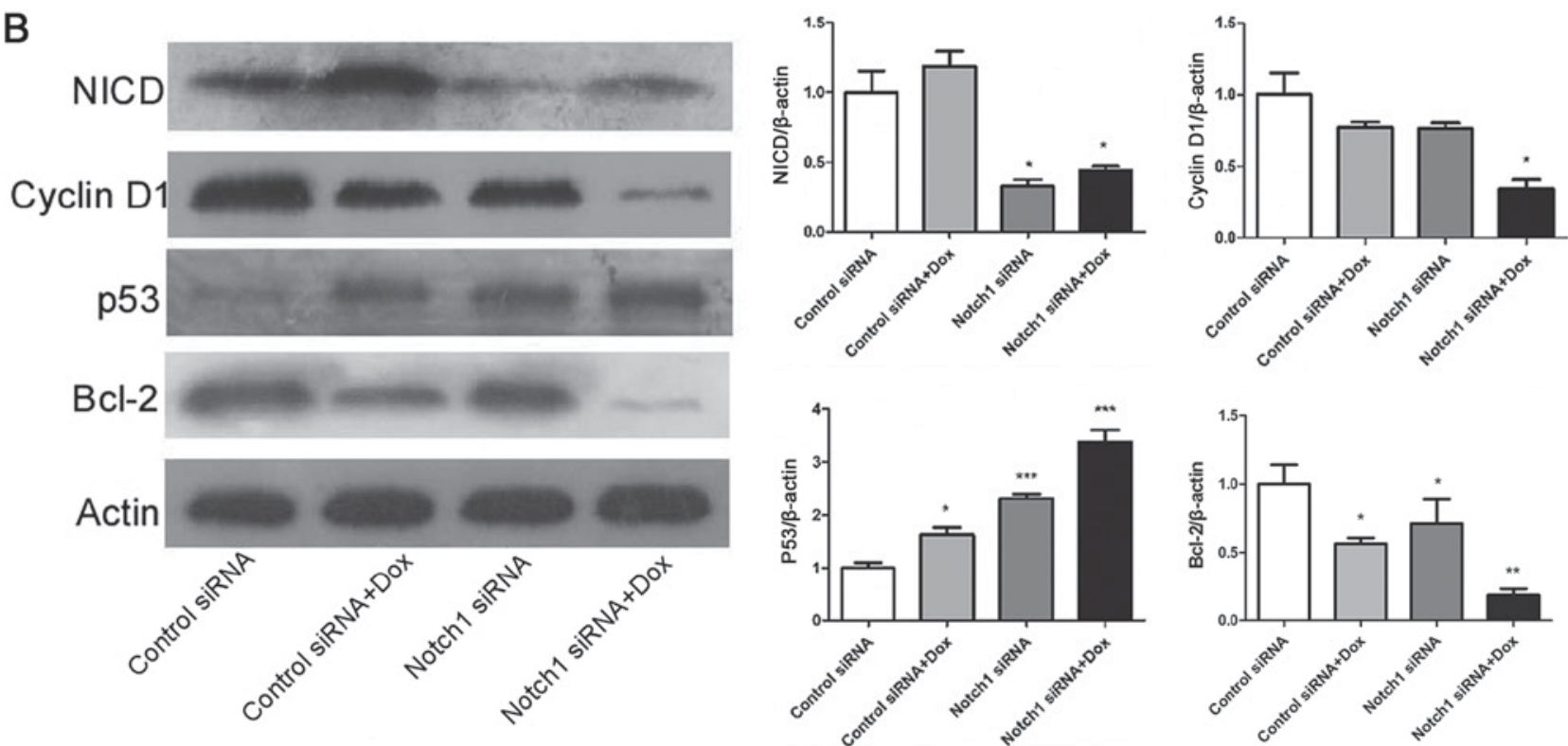

Figure 3. Combined effect of Notch1 siRNA and doxorubicin on gastric cancer cell apoptosis. (A) The apoptosis was measured by flow cytometer. (B) Notch1, NICD and apoptosis-associated proteins were determined by western blotting. (C) Analysis of relative caspase-3 activity in $\mathrm{SGC} 7901$. ${ }^{*} \mathrm{P}<0.05,{ }^{* *} \mathrm{P}<0.01$, ${ }^{* * * *} \mathrm{P}<0.001$ vs. control siRNA or as indicated. NICD, Notch1 intracellular domain; p53, tumor protein p53; Bcl-2, B-cell lymphoma-2; siRNA, small interfering RNA; Dox, doxorubicin.

Efficacy and mechanism of action of doxorubicin combined with Notchl siRNA on GC in vitro. Previous results in the present study revealed that combination treatment with doxorubicin and Notch1 increased the sensitivity of GC to doxorubicin. The present study examined whether the increase in doxorubicin sensitivity was associated with Notch1-induced apoptosis. Levels of apoptosis in control siRNA-transfected cells were similar to or slightly reduced $(4.1 \pm 1.2 \%)$ compared with those of untransfected cells. By contrast, the combination of Notch1 siRNA with doxorubicin $(32.1 \pm 2.9 \%)$ significantly promoted apoptosis compared to doxorubicin $(14.1 \pm 2.7 \%)$ or Notch1 siRNA $(15.1 \pm 3.1 \%)$ treatment alone (Fig. 3A). Next, the expression of cell cycle effectors and apoptosis-associated proteins was examined (Fig. 3A). Doxorubicin did not affect NICD protein levels, whereas doxorubicin and Notch1 siRNA had similar effects on cyclin D1. Treatment with Notch1
siRNA exerted stronger inhibitory effects on p53 expression than treatment with doxorubicin, whereas doxorubicin exerted stronger inhibitory effects on Bcl-2 expression (Fig. 3B). In addition, the combination of Notch1 siRNA and doxorubicin had the greatest effects on the apoptosis of GC cells. Caspase-3 activity in SGC7901 cells treated with doxorubicin and Notch1 siRNA was markedly higher than that in SGC7901 cells treated with doxorubicin or Notch1 siRNA alone (Fig. 3C).

Efficacy of doxorubicin combined with Notch1 siRNA in vivo. The tumor volumes in the nude mice in the three test groups were measured within 32 days after xenogenic GC cell transplantation (Fig. 4A). The average tumor volume in the Notch1 siRNA group was markedly lower than that of the control siRNA group. As expected, there were no major differences between the control siRNA and SGC7901 groups. Apoptotic 
A

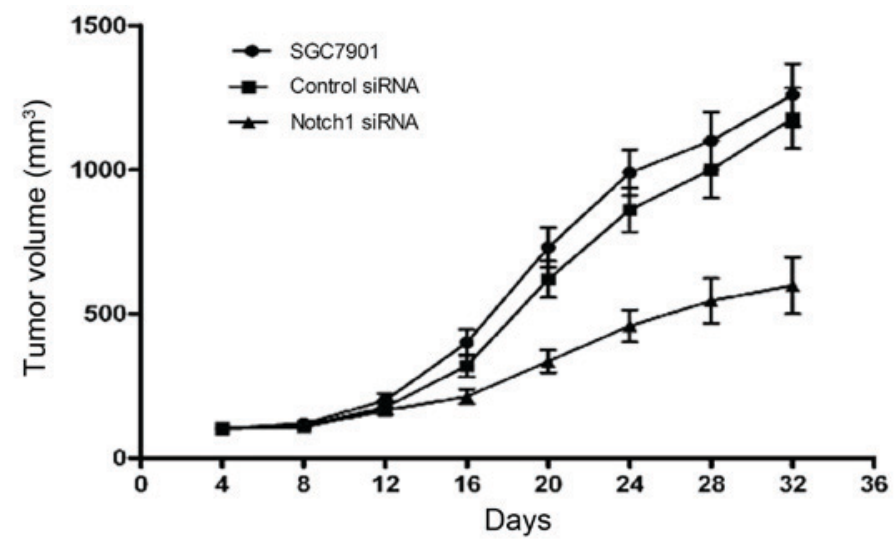

B

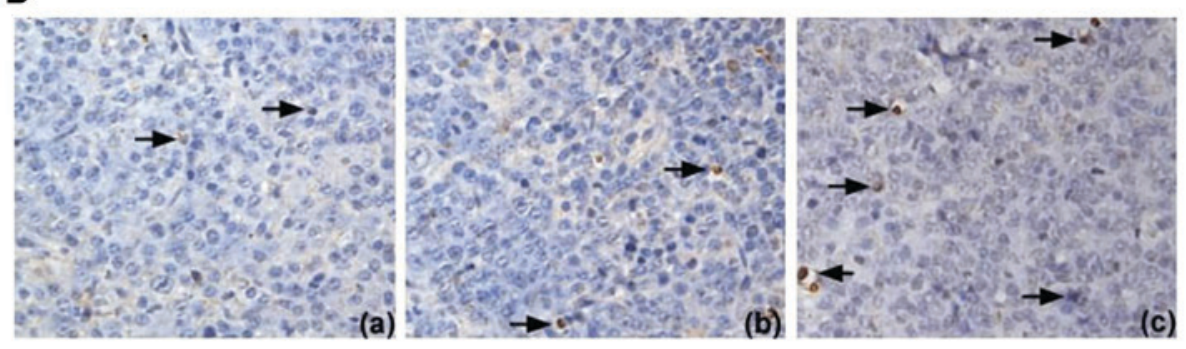

Figure 4. Combined effect of Notch1 siRNA and doxorubicin on tumor growth suppression in vivo. (A) Tumor size and tumor volume of nude mice in each group (SGC7901, control siRNA, Notch1 siRNA). (B) Identification of apoptotic cells in tumor tissues was performed using the terminal deoxynucleotidyl transferase dUTP nick-end labeling assay (magnification, x400). (Ba) Tumors from the SGC7901 group; (Bb) tumors from control siRNA; (Bc) tumors from Notch1 siRNA. The brown color designating the apoptotic signals is indicated by the arrows. siRNA, small interfering RNA.

bodies in subcutaneous tumor sections were detected by TUNEL staining (Fig. 4B). The results demonstrated that the rate of apoptosis in the Notch1 siRNA group $(31.7 \pm 3.9 \%)$ was significantly higher than that in control siRNA $(12.7 \pm 3.2 \%)$ and SGC7901 (11.6 $\pm 2.9 \%)$ groups.

\section{Discussion}

The role of the Notch1 signaling pathway is complicated and its regulatory mechanism is not completely understood (23). A previous study demonstrated that Notch1 was highly expressed in GC, and that Notch1 expression promoted tumorigenesis through a variety of signaling pathways (24). In addition, Notch1 activation is considered to be a novel independent prognostic factor for GC (25). The present study revealed that Notch1 activation levels, measured as levels of NICD, were high in GC tissues and cell lines. On the basis of the results of the in vitro Notch1 depletion experiments, transfection of Notch1 siRNA significantly decreased Notch1 and NICD levels, which dramatically inhibited the apoptosis of SGC7901 cells. These findings are consistent with the results of previous investigations. The present study examined SGC7901 cells only; more gastric cancer cell lines are necessary to be used to confirm these results in future studies.

Cell cycle initiation and progression are regulated by several classic cyclins, including cyclin D1 (26). Cyclin D1 is a marker of cellular proliferation and is reported to be significantly elevated in GC (27). In the present study, decreased Notch1 levels were accompanied by decreased cyclin D1 levels. In addition, $\mathrm{Bcl}-2$ expression was also significantly decreased, whereas that of p53 exhibited an opposite pattern of expression. Under these conditions, caspase-3 activity exhibited a marked rise. p53 is a critical tumor suppressor gene that negatively regulates a number of apoptosis activators, including Bcl-2 (28). Although Bcl-2 expression was decreased, caspase-3, a key apoptotic molecule, was activated when the cells began to undergo apoptosis (29). Therefore, the data from the current study indicated that downregulation of NICD by Notch1 siRNA downregulates the p53-dependent pathway and induces apoptosis in GC.

Doxorubicin is a commonly used chemotherapy drug with proven pharmacological effects; however, its use in GC is limited by serious side effects and frequent resistance $(30,31)$. To the best of our knowledge, the mechanism of drug resistance in cancer is complicated and remains unclear. It has been reported that the Wnt/3-catenin (32), phosphoinositide 3-kinase/RAC serine/threonine-protein kinase (Akt)/mechanistic target of rapamycin (mTOR) (33) and Hippo (34) pathways are associated with drug resistance in different cancer types. There are certain pathways being investigating for doxorubicin resistance, including the Akt/mTOR (35), dual specificity phosphase 4 (36), G protein-coupled receptor 81 (37) and numerous miRNA (38) pathways. Thus, further prospective studies are required to investigate other molecular-targeted drugs associated with doxorubicin resistance. In the present experiment, depletion of NICD by Notch1 siRNA significantly increased the chemosensitivity of SGC7901 cells to doxorubicin, decreased cell proliferation and increased the rate of apoptosis. Additionally, in the xenograft tumor-bearing nude mouse model, combination therapy with Notch1 siRNA and doxorubicin was more effective than doxorubicin alone. These data indicated that the combination of doxorubicin and Notch1 siRNA may represent a potential therapeutic approach for GC. It was previously revealed that a number of genes, 
such as MRP-1 (16) and epithelial to mesenchymal transition (EMT) (39) were the downregulated genes of Notch1 for the drug resistance. In the present study, the expression of p53 protein was more potently upregulated by Notch1 siRNA than by doxorubicin, whereas Bcl-2 expression gave opposite results. Previous studies demonstrated that doxorubicin could induce tumor apoptosis via p53 pathways (4). These results indicated that Notch1 siRNA could enhance doxorubicin-induced apoptosis by activating the p53 pathway.

In conclusion, inhibition of Notch1 with a Notch1 siRNA could increase the sensitivity of GC cells to doxorubicin in vitro and in vivo. Thus, Notch1 is an excellent target for chemotherapy with doxorubicin, indicating an appropriate combination of doxorubicin and downregulation of Notch1 expression as a potential future strategy for the treatment of human GC.

\section{Acknowledgements}

The authors thank Mr Hong Xia from Wuhan University (Wuhan, China) for his outstanding administrative support in this work.

\section{Funding}

Funding was received from the Natural Science Foundation of China (grant no. 81401959).

\section{Availability of data and materials}

All data generated or analyzed during the present study are included in this published article.

\section{Authors' contributions}

WZ and HGY designed the experiment, WZ, WT and XH performed the experiments, WZ wrote the manuscript and WZ, WT and XH analyzed the data.

\section{Ethics approval and consent to publish}

All animal procedures were approved by the Ethical Committee on Animal Experimentation of Renmin Hospital of Wuhan University [WDRM (FU) approval no. 20151012]. All human tissue procedures were approved by the Ethical Committee on Clinical Research of Renmin Hospital of Wuhan University (approval no. AF SOP/3.6-01/5.1). Written informed consent was obtained from all patients.

\section{Consent for publication}

Not applicable.

\section{Competing interests}

The authors declare that they have no competing interests.

\section{References}

1. Ferlay J, Soerjomataram I, Dikshit R, Eser S, Mathers C, Rebelo M, Parkin DM, Forman D and Bray F: Cancer incidence and mortality worldwide: Sources, methods and major patterns in GLOBOCAN 2012. Int J Cancer 136: E359-E386, 2015.
2. Kang Y, Li S, Ge Q, Liu X, Yang Z, Xue Y and Wang F: Extent of serosal changes predicts peritoneal recurrence and poor prognosis after curative surgery for gastric cancer. Medicine (Baltimore) 94: e1750, 2015.

3. Wagner AD, Grothe W, Haerting J, Kleber G, Grothey A and Fleig WE: Chemotherapy in advanced gastric cancer: A systematic review and meta-analysis based on aggregate data. J Clin Oncol 24: 2903-2909, 2006.

4. Tacar O, Sriamornsak P and Dass CR: Doxorubicin: An update on anticancer molecular action, toxicity and novel drug delivery systems. J Pharm Pharmacol 65: 157-170, 2013.

5. Xu J, Liu D, Niu H, Zhu G, Xu Y, Ye D, Li J and Zhang Q: Resveratrol reverses Doxorubicin resistance by inhibiting epithelial-mesenchymal transition (EMT) through modulating PTEN/Akt signaling pathway in gastric cancer. J Exp Clin Cancer Res 36: 19, 2017.

6. Zhou Q, Wang Y, Peng B, Liang L and Li J: The roles of Notch1 expression in the migration of intrahepatic cholangiocarcinoma. BMC Cancer 13: 244, 2013

7. Ke Z, Mao X, Li S, Wang R, Wang L and Zhao G: Dynamic expression characteristics of Notch signal in bone marrow-derived mesenchymal stem cells during the process of differentiation into hepatocytes. Tissue Cell 45: 95-100, 2013.

8. Qiu H, Tang X, Ma J, Shaverdashvili K, Zhang K and Bedogni B: Notch1 autoactivation via transcriptional regulation of furin, which sustains Notch1 signaling by processing Notch1-activating proteases ADAM10 and membrane type 1 matrix metalloproteinase. Mol Cell Biol 35: 3622-32, 2015.

9. Soylu H, Acar N, Ozbey O, Unal B, Koksal IT, Bassorgun I, Ciftcioglu A and Ustunel I: Characterization of Notch signalling pathway members in normal prostate, prostatic intraepithelial neoplasia (PIN) and prostatic adenocarcinoma. Pathol Oncol Res 22: 87-94, 2016.

10. Colomer-Lahiguera S and Strehl S: Complexity of NOTCH1 juxtamembrane insertion mutations in T-cell acute lymphoblastic leukemia. Leuk Lymphoma 57: 1219-1222, 2016.

11. Rodriguez JM, Miranda D, Bunout D, Ronco AM, de la Maza MP and Hirsch S: Folates induce colorectal carcinoma HT29 cell line proliferation through Notch1 signaling. Nutr Cancer 67: 706-711, 2015.

12. Hassan WA, Yoshida R, Kudoh S, Hasegawa K, Niimori-Kita K and Ito T: Notch1 controls cell invasion and metastasis in small cell lung carcinoma cell lines. Lung Cancer 86: 304-310, 2014.

13. Yeh TS, Wu CW, Hsu KW, Liao WJ, Yang MC, Li AF, Wang AM, Kuo ML and Chi CW: The activated Notch1 signal pathway is associated with gastric cancer progression through cyclooxygenase-2. Cancer Res 69: 5039-5048, 2009.

14. Harada K, Sato Y, Ikeda H, Hsu M, Igarashi S and Nakanuma Y: Notch1-Hes1 signalling axis in the tumourigenesis of biliary neuroendocrine tumours. J Clin Pathol 66: 386-391, 2013.

15. Pedrosa AR, Graça JL, Carvalho S, Peleteiro MC, Duarte A and Trindade A: Notch signaling dynamics in the adult healthy prostate and in prostatic tumor development. Prostate 76: 80-96, 2016.

16. Hassan WA, Yoshida R, Kudoh S, Kameyama H, Hasegawa K, Niimori-Kita K and Ito T: Notch1 controls cell chemoresistance in small cell lung carcinoma cells. Thorac Cancer 7: 123-128, 2016.

17. Lian W, Zhang L, Yang L and Chen W: AP- $2 \alpha$ reverses vincristine-induced multidrug resistance of SGC7901 gastric cancer cells by inhibiting the Notch pathway. Apoptosis 22: 933-941, 2017.

18. Zhou W, Fu XQ, Zhang LL, Zhang J, Huang X, Lu XH, Shen L, Liu BN,Liu J,LuoHS, et al: The AKT1/NF-kappaB/Notch1/PTEN axis has an important role in chemoresistance of gastric cancer cells. Cell Death Dis 4: e847, 2013.

19. Zhang XS, Hu YH, Gao HY, Lan XW and Xue YW: Downregulation of Notch1 inhibits the invasion and metastasis of human gastric cancer cells SGC7901 and MKN74 in vitro through PTEN activation and dephosphorylation of Akt and FAK. Mol Med Rep 16: 2318-2324, 2017.

20. World Medical Association: World Medical Association Declaration of Helsinki: ethical principles for medical research involving human subjects. JAMA 310: 2191-2194, 2013.

21. Wang M, Chen DQ, Chen L, Liu D, Zhao H, Zhang ZH, Vaziri ND, Guo Y, Zhao YY and Cao G: Novel RAS inhibitors poricoic acid $\mathrm{ZG}$ and poricoic acid $\mathrm{ZH}$ attenuate renal fibrosis via $\mathrm{Wnt} / \beta$-catenin pathway and targeted phosphorylation smad3 signaling. J Agric Food Chem 66: 1828-1842,2018. 
22. National Research Council, Committee for the Update of the Guide for the Care and Use of Laboratory, Institute for Laboratory Animal Research and Division on Earth and Life Studies: Guide for the Care and Use of Laboratory Animals, 8th edition. The National Academies Press, Washington, DC, 2011.

23. Fender AW, Nutter JM, Fitzgerald TL, Bertrand FE and Sigounas G: Notch-1 promotes stemness and epithelial to mesenchymal transition in colorectal cancer. J Cell Biochem 116 2517-2527, 2015.

24. Hsu KW, Hsieh RH, Huang KH, Fen-Yau Li A, Chi CW, Wang TY, Tseng MJ, Wu KJ and Yeh TS: Activation of the Notch1/STAT3/Twist signaling axis promotes gastric cancer progression. Carcinogenesis 33: 1459-1467, 2012.

25. Zhang $\mathrm{H}$, Wang $\mathrm{X}, \mathrm{Xu} \mathrm{J}$ and Sun Y: Notch1 activation is a poor prognostic factor in patients with gastric cancer. Br J Cancer 110: 2283-2290, 2014.

26. Villasmil ML, Francisco J, Gallo-Ebert C, Donigan M, Liu HY, Brower $\mathrm{M}$ and Nickels JT Jr: Ceramide signals for initiation of yeast mating-specific cell cycle arrest. Cell Cycle 15: 441-454, 2016.

27. Seo JH, Jeong ES and Choi YK: Therapeutic effects of lentivirus-mediated shRNA targeting of cyclin D1 in human gastric cancer. BMC Cancer 14: 175, 2014.

28. Vadde R, Radhakrishnan S, Reddivari L and Vanamala JK: Triphala extract suppresses proliferation and induces apoptosis in human colon cancer stem cells via Suppressing c-Myc/Cyclin D1 and elevation of $\mathrm{Bax} / \mathrm{Bcl}-2$ ratio. Biomed Res Int 2015: 649263, 2015.

29. Liu E, Liang T, Wang X, Ban S, Han L and Li Q: Apoptosis induced by farrerol in human gastric cancer SGC-7901 cells through the mitochondrial-mediated pathway. Eur J Cancer Prev 24: 365-372, 2015

30. Choi JH, Lim HY, Joo HJ, Kim HS, Yi JW, Kim HC, Cho YK, Kim MW and Lee KB: Expression of multidrug resistance-associated protein1, P-glycoprotein, and thymidylate synthase in gastric cancer patients treated with 5-fluorouracil and doxorubicin-based adjuvant chemotherapy after curative resection. Br J Cancer 86: 1578-1585, 2002.
31. Yu HG, Ai YW, Yu LL, Zhou XD, Liu J, Li JH, Xu XM, Liu S, Chen J, Liu F, et al: Phosphoinositide 3-kinase/Akt pathway plays an important role in chemoresistance of gastric cancer cells against etoposide and doxorubicin induced cell death. Int J Cancer 122: 433-443, 2008

32. Cui J, Jiang W, Wang S, Wang L and Xie K: Role of Wnt/ $\beta$-catenin signaling in drug resistance of pancreatic cancer. Curr Pharm Des 18: 2464-2471, 2012

33. Tang KD and Ling MT: Targeting drug-resistant prostate cancer with dual PI3K/mTOR inhibition. Curr Med Chem 21: 3048-3056, 2013.

34. Zhao Y and Yang X: The Hippo pathway in chemotherapeutic drug resistance. Int J Cancer 137: 2767-2773, 2015.

35. Broxterman HJ, Gotink KJ and Verheul HM: Understanding the causes of multidrug resistance in cancer: A comparison of doxorubicin and sunitinib. Drug Resist Updat 12: 114-126, 2009.

36. Kang X, Li M, Zhu H, Lu X, Miao J, Du S, Xia X and Guan W: DUSP4 promotes doxorubicin resistance in gastric cancer through epithelial-mesenchymal transition. Oncotarget 8: 94028-94039, 2017.

37. Wagner W, Kania KD, Blauz A and Ciszewski WM: The lactate receptor (HCAR1/GPR81) contributes to doxorubicin chemoresistance via ABCB1 transporter up-regulation in human cervical cancer HeLa cells. J Physiol Pharmacol 68: 555-564, 2017.

38. Rizzo S, Cangemi A, Galvano A, Fanale D, Buscemi S, Ciaccio M, Russo A, Castorina S and Bazan V: Analysis of miRNA expression profile induced by short term starvation in breast cancer cells treated with doxorubicin. Oncotarget 8: 71924-71932, 2017.

39. Kotiyal S and Bhattacharya S: Breast cancer stem cells, EMT and therapeutic targets. Biochem Biophys Res Commun 453: 112-116, 2014.

c) (i) $(9)$ This work is licensed under a Creative Commons

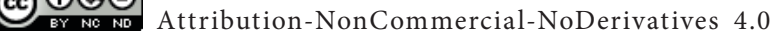
International (CC BY-NC-ND 4.0) License. 